\title{
(Self-)similar groups and the Farrell-Jones conjectures
}

\author{
Laurent Bartholdi*
}

\begin{abstract}
We show that contracting self-similar groups satisfy the Farrell-Jones conjectures as soon as their universal contracting cover is non-positively curved. This applies in particular to bounded self-similar groups.

We define, along the way, a general notion of contraction for groups acting on a rooted tree in a not necessarily self-similar manner.
\end{abstract}

Mathematics Subject Classification (2010). 20E08, 19D10, 20 F65.

Keywords. Self-similar bounded group, K-theory.

\section{Introduction}

Few properties are known to hold for all groups; in the recent years, counterexamples have been found to numerous "plausible conjectures", usually formulated as questions: is there an infinite, finitely generated group all of whose elements have finite order? is there an amenable group that cannot be produced using extensions and filtered colimits of virtually abelian groups? is there a group whose word growth is strictly between polynomial and exponential?

The "Farrell-Jones conjectures", predicting how the algebraic K-/L-theory of the group ring $R G$ may be expressed in terms of the algebraic $\mathrm{K}$-/L-theory of $R$ and the group theory of $G$, is one of the prominent remaining conjectures [7]. If it is satisfied by the group $G$, numerous group-theoretical consequences for $G$ follow, in particular $R G$ has no non-trivial idempotents if $G$ is torsion-free and $R$ is a domain of characteristic 0 . The Farrell-Jones conjectures are inherited under many grouptheoretical operations (finite direct and free products, filtered colimits), but possibly not under wreath products; we say the Farrell-Jones conjectures hold with wreathing if they hold for all wreath products $G<P$ with a finite permutation group $P$.

In search of a possible counterexample to the Farrell-Jones conjectures, it might have been speculated that the "self-similar groups" studied by Alëshin, Grigorchuk,

\footnotetext{
*The work is supported by the Courant Research Centre "Higher Order Structures" of the University of Göttingen.
} 
Gupta and Sidki since the 1970s would play an important role; indeed, these groups have served to answer or illuminate all the questions in the first paragraph.

Self-similar groups are groups acting in a recursive manner on a regular rooted tree $T_{d}$. If the recursion of every element involves only a linearly growing subtree of $T_{d}$, the group is said to be bounded.

We show in this note that, if it is at all possible, considerable care will be required to construct a counterexample within the class of self-similar groups. We prove (see below for precise definitions):

Theorem A. Let $G$ be a bounded self-similar group. Then $G$ satisfies the FarrellJones conjectures.

Theorem B. Let $G$ be a contracting similar group. Then $G$ satisfies the Farrell-Jones conjectures if its universal contracting cover satisfies the Farrell-Jones conjectures with wreathing.

Corollary C. The Alëshin, Grigorchuk, Gupta-Sidki, GGS, and generalized Grigorchuk groups all satisfy the Farrell-Jones conjectures.

Acknowledgments. Wolfgang Lück encouraged me to write this short note, with the intent of narrowing the domains of group theory in which a counterexample is to be searched.

Thomas Schick and Wolfgang Lück generously provided valuable feedback on a preliminary version, and clarified for me the status of the Farrell-Jones conjectures with respect to wreath products.

\section{The Farrell-Jones conjectures}

We review very briefly the statement of the Farrell-Jones conjectures; we include them for definiteness but will never work directly with their definition.

A model for the virtually cyclic classifying space $E^{\mathrm{vc}}(G)$ is a topological $G$-space $X$ whose isotropy groups are all virtually cyclic, and such that for any topological $G$-space $Y$ with virtually cyclic isotropy groups there exists up to $G$-homotopy a unique $G$-map $Y \rightarrow X$.

The Farrell-Jones conjectures assert that the natural map

$$
H_{n}^{G}\left(E^{\mathrm{vc}}(G), \mathbf{S}\right) \rightarrow H_{n}^{G}(\{.\}, \mathbf{S}),
$$

induced by $E^{\mathrm{vc}}(G) \rightarrow\{$.$\} , is an isomorphism for all n$. Here $\mathbf{S}$ is either the K-theory spectrum $\mathbf{K}_{\mathcal{A}}$ or the L-theory spectrum $\mathbf{L}_{\mathcal{A}}^{\langle-\infty\rangle}$ over the orbit category associated with an additive $G$-category $\mathcal{A}$.

For our purposes, it suffices to note that the class of groups for which the conjectures are known to hold contains virtually abelian groups, hyperbolic groups [6] 
for $n \leq 1$, CAT(0) groups [6], [28], cocompact lattices in virtually connected Lie groups, threefold groups [4] and arithmetic groups over algebraic number fields (unpublished). It is closed under taking subgroups, colimits (Corollary 0.8 of [5]), and finite direct and free products. (This is the advantage of using the more general version with coëfficients in an additive category - the inheritance properties come almost for free.)

Note that, in general, it is not known whether the conjectures are inherited under finite extensions. Since every finite extension is a subgroup of the wreath product with a finite group [23], the question reduces to whether the conjecture is inherited by finite wreath products. This is known in some specific cases, in particular for cocompact lattices in virtually connected Lie groups, threefold groups, arithmetic groups over algebraic number fields, and CAT(0) groups, as we now explain.

CAT(0) spaces are metric spaces in which triangles are at least as thin as in euclidean space; see the classical reference [13]. CAT(0) groups, also called nonpositively curved groups, are groups acting properly, isometrically and cocompactly on a CAT(0) space of finite topological dimension. That class contains virtually abelian groups, and is closed under direct, free and finite wreath products.

Lemma 1. If $G$ is $\mathrm{CAT}(0)$, then so is $G$ ? $P$ for any finite permutation group $P$.

Proof. Let $G$ act properly discontinuously on the CAT(0) space $X$, and let $P$ be a permutation group on $n$ points. Then $G \imath P=G^{n} \rtimes P$ acts properly discontinuously on $X^{n}$, with $G^{n}$ acting coördinatewise and $P$ by permutation of the coördinates.

\section{3. (Self-)similar groups}

We summarize the notion of self-similar group, presenting it in a slightly more general and algebraic manner than is usual; see [25] or [8] for classical references. By $G<d$ we denote the permutational wreath product $G^{d} \rtimes \mathfrak{S}_{d}$ with the symmetric group on $d$ letters.

A self-similar group is a group $G$ endowed with a homomorphism $\phi: G \rightarrow G \nmid d$, called its self-similarity structure. The integer $d$ is the degree of the self-similarity structure. Usually, the self-similarity is implicit, and one simply denotes by $G$ the self-similar group.

The map $\phi$ can be applied diagonally to all entries in $G^{d}$, yielding a map $G^{d} \rightarrow$ $(G<d)^{d}$, and therefore a map $G<d \rightarrow(G<d)<d \subseteq G<\left(d^{2}\right)$; more generally, we get maps $G<d^{n} \rightarrow G<d^{n+1}$ which we all denote by $\phi$. We may compose these maps, and write $\phi^{n}$ for the iterate $\phi^{n}: G \rightarrow G<d^{n}$.

By projecting to the permutation part, we have homomorphisms $G \rightarrow \mathfrak{S}_{d^{n}}$ and, assembling them together, a permutational action of $G$ on $T_{d}:=\bigsqcup_{n \geq 0}\{1, \ldots, d\}^{n}$; one may identify $T_{d}$ with the vertex set of a rooted $d$-regular tree, by connecting $v_{1} \ldots v_{n}$ to $v_{1} \ldots v_{n} v_{n+1}$ for all $v_{i} \in\{1, \ldots, d\}$, in such a way that $G$ acts by 
graph isometries. This action need not be faithful; if it is, then $G$ is called a faithful self-similar group.

A self-similar group is contracting if there exists a finite subset $N \subset G$ such that, for all $g \in G$ and all $n$ large enough, $\phi^{n}(g) \in N^{d^{n}} \times \widetilde{S}_{d^{n}}$. The smallest such $N$ is called the nucleus of $G$.

Let $\widetilde{F}$ denote the free group on $N$. By definition, the nucleus satisfies the condition $\phi(N) \subset N^{d} \times \subseteq_{d}$. The restriction of $\phi$ to $N$ can therefore uniquely be extended to a homomorphism $\tilde{\phi}: \widetilde{F} \rightarrow \widetilde{F}$ ?d. Set

$$
R=\left\{w \in N \cup N^{2} \cup N^{3} \subset \widetilde{F} \mid w={ }_{G} 1\right\} .
$$

Similarly, we have $\tilde{\phi}(R) \subset R^{d} \times 1$. Set $F=\tilde{F} / R$. The homomorphism $\tilde{\phi}$ then induces a homomorphism again written $\phi: F \rightarrow F<d$.

Note that $F$ is a finitely presented group, and that the natural map $N \subset \widetilde{F} \rightarrow N \subset$ $G$ defines a homomorphism $F \rightarrow G$. We will see in Lemma 2 that $F$ is contracting, with nucleus $N$. However, the self-similarity structure of $F$ need not be faithful, even if that of $G$ was faithful. We call $F$ the universal contracting cover of $G$. Note also that in general the homomorphism $F \rightarrow G$ need not be onto, or equivalently $N$ need not generate $G$. This is, however, the case in all examples we present here.

Here are some extreme examples; more classical ones appear in $\S 4$. The full group $W$ of isometries of $T_{d}$ is self-similar, but not contracting; actually not even countable. Its subgroup $\left\{g \in W \mid \psi^{n}(g) \in\{1\}^{d^{n}} \times \mathbb{S}_{d^{n}}\right.$ for some $\left.n\right\}$ is faithful, self-similar, and contracting with nucleus $\{1\}$. Any group $G$, with $\phi: G \rightarrow G^{d}$ the diagonal embedding, defines a non-faithful self-similar structure on $G$, which is contracting precisely when $G$ is finite. Consider finally $A$ a finite group, and $G$ the group of finitelysupported functions $\mathbb{Z} \rightarrow A$. Take $d=2$, and set $\psi(f)=\left\langle\left\langle f_{0}, f_{1}\right\rangle\right\rangle$ with $f_{0}(n)=$ $f(2 n)$ and $f_{1}(n)=f(2 n-1)$. This defines a self-similarity structure on $G$, which is not faithful, and contracting with nucleus $N=$ functions supported on $\{0,1\}\}$. Our main result does not give any interesting information on such actions.

3.1. Similar groups. We now extend the definitions above to more general groups. A group $G$ is similar if there exists a sequence $G=G_{0}, G_{1}, \ldots$ of groups, a sequence of integers $d_{1}, d_{2}, \ldots$, and a sequence of homomorphisms $\phi_{n}: G_{n} \rightarrow$ $G_{n+1} \prec d_{n+1}$. The similarity structure is faithful if the corresponding permutational action on $\bigsqcup_{n>0}\left\{1, \ldots, d_{1}\right\} \times \cdots \times\left\{1, \ldots, d_{n}\right\}$ is faithful. Again abusing notation, the compositions of $\phi_{n}$ 's are written $\phi_{n}^{m}: G_{n} \rightarrow G_{n+m} \nmid d_{n+1} d_{n+2} \ldots d_{n+m}$.

Let $N_{0}, N_{1}, \ldots$ be a sequence of finite sets, with $N_{n} \subset G_{n}$ for all $n$. We say that $G$ contracts to $\left(N_{n}\right)_{n \geq 0}$ if for every $g \in G_{n}$ and every $m$ large enough, $\phi_{n}^{m}(g) \in$ $N_{n+m}^{d_{n+1} d_{n+2} \ldots d_{n+m}} \times \widetilde{\subseteq}_{d_{n+1} d_{n+2} \ldots d_{n+m}}$.

In that case, it is possible, up to enlarging the $N_{n}$ 's, to assume $\phi_{n}\left(N_{n}\right) \subset$ $N_{n+1}^{d_{n+1}} \times \Im_{d_{n+1}}$, and we always make that additional assumption. We call the sequence $N_{0}, N_{1}, \ldots$ a nucleus of $G$. 
Note however that the sequence $N_{0}, N_{1}, \ldots$ is not unique - for example, it is always possible to replace finitely many of the initial terms by 1 . We say $G$ is generated by the nucleus if $N_{n}$ generates $G_{n}$ for all $n$.

Extending the previous definition, let $F_{n}$ be the finitely presented group

$$
\left.F_{n}:=\left\langle N_{n}\right| \text { words of length } \leq 3 \text { that are } \equiv 1 \text { in } G_{n}\right\rangle
$$

We then have induced homomorphisms $F_{n} \rightarrow F_{n+1}<d_{n+1}$, defining a similarity structure for the group $F:=F_{0}$.

Lemma 2. The similar group $F$ contracts to $\left(N_{n}\right)_{n \geq 0}$.

We again call $F$ the universal contracting cover of $G$; note that it depends on the choice of $\left(N_{n}\right)_{n \geq 0}$.

Proof. Consider $n \in \mathbb{N}$. For every $g \in N_{n}^{\leq 2} \subset G_{n}$, there exists $m \in \mathbb{N}$ such that $\phi_{n}^{m}(g) \in N_{n+m}^{d_{n+1} d_{n+2} \ldots d_{n+m}} \times \Im_{d_{n+1} d_{n+2} \ldots d_{n+m}}$, by the contraction condition. Since there are finitely many $g$ 's under consideration, there exists $m_{n} \in \mathbb{N}$ such that

$$
\phi_{n}^{m_{n}}\left(N_{n}^{2}\right) \in N_{n+m_{n}}^{d_{n+1} d_{n+2} \ldots d_{n+m_{n}}} \times \Im_{d_{n+1} d_{n+2} \ldots d_{n+m_{n}}} .
$$

On the other hand, consider $\tilde{w} \in \widetilde{F}_{n}$ a word of length $\ell \leq 2$ in the alphabet $N_{n}$, and denote by $\tilde{w}$ and $w$ respectively its image in $G_{n}$ and in $F_{n}$. The entries in $\tilde{\phi}_{n}^{m_{n}}(\tilde{w})$ have length precisely $\ell$, by construction. They are termwise equal, in $G_{n+m_{n}}$, to the entries of $\phi_{n}^{m_{n}}(\tilde{w})$. Since $F_{n+m_{n}}$ contains all relations of length $\leq 3$, these entries are also termwise equal in $F_{n+m_{n}}$. It follows that, for every $w \in F_{n}$ of length $\leq 2$, all entries of $\phi_{n}^{m_{n}}(w)$ all belong to $N_{n+m_{n}}$.

Consider now $g \in F_{n}$, of length $\ell \leq 2^{k}$ in the alphabet $N_{n}$. Set inductively $n_{0}=n$ and $n_{i+1}=n_{i}+m_{n_{i}}$. By the previous paragraph, the entries of $\phi_{n}^{m_{n}}(g)$ have length $\leq 2^{k-1}$ over $N_{n+m_{n}}=N_{n_{1}}$, and more generally the entries of $\phi_{n}^{n_{k}-n}$ have length $\leq 2^{0}$ in $N_{n_{k}}$, that is, they belong to $N_{n_{k}}$.

We call a similar group contracting if it has been endowed with a sequence $\left(N_{n}\right)_{n \geq 0}$ to which it contracts. Note that this fixes the choice of a contracting finitely presented cover. Similar contracting groups naturally include self-similar groups, by considering constant sequences $G, \phi, N$ and $F$.

Note that we explicitly allow the sequences $G, \phi$ to be constant while the $N_{n}$ 's increase. Quite generally, if each $G_{n}$ is countable, then there exists a sequence of finite sets to which it contracts; namely, enumerate $G_{n}=\left\{g_{n, 1}, g_{n, 2}, \ldots\right\}$, and let $N_{n}$ be the set of coördinates of $\phi_{m}^{n-m}\left(g_{m, i}\right)$ for all $i, m \leq n$. Understandably, our main result applies formally to such constructions but does not yield any useful information. 


\subsection{Main result}

Proposition 1. Let G be a faithful contracting similar group, generated by its nucleus. If all terms $F_{n}$ of the universal contracting cover of $G$ satisfy the Farrell-Jones conjectures with wreathing, then $G$ satisfies the Farrell-Jones conjectures.

Proof. In the self-similar case, set $K_{0}=1 \triangleleft F$, and $K_{n+1}=\phi^{-1}\left(K_{n}^{d}\right)$ for all $n \geq 0$; and finally $K_{\infty}=\bigcup_{n \geq 0} K_{n}$. More generally, in the similar case, set $K_{n}=$ $\operatorname{ker}\left(\phi^{n}\right) \triangleleft F$ and $K_{\infty}=\bigcup_{n \geq 0} K_{n}$.

There is a natural homomorphism $\pi: F / K_{\infty} \rightarrow G$, which we prove to be an isomorphism. Let $g \in F$ be in the kernel of $\pi$; then, because $F$ is contracting, there is $n \in \mathbb{N}$ such that $\phi^{n}(g)$ belongs to $N_{n}^{d_{1} \ldots d_{n}} \times \widetilde{S}_{d_{1} \ldots d_{n}}$; furthermore, the permutation is trivial because $\phi^{n} \pi(g)=\phi^{n}(1)=1$, and the entries in $N_{n}$ are trivial because $F_{n}$ contains relations of length 1 in $N_{n}$. Therefore $g \in K_{n}$, so $g \in K_{\infty}$, as was to be shown.

We then have $G=\lim F / K_{n}$, and because the Farrell-Jones conjectures are stable under colimits it suffices to see that $F / K_{n}$ satisfies the Farrell-Jones conjectures. By the first isomorphism theorem, $F / K_{n}$ is a subgroup of $F_{n} \prec d_{1} \ldots d_{n}$, so it suffices to show that $F_{n} \succ d_{1} \ldots d_{n}$ satisfies the Farrell-Jones conjectures. Since $F_{n}$ satisfies the Farrell-Jones conjectures with wreathing, we are done.

As stated in the introduction, Proposition 1 applies in particular to contracting similar groups whose universal contracting cover are CAT(0) groups, lattices in virtually connected Lie groups, or arithmetic groups over algebraic function fields.

\section{Examples}

We now give some examples of contracting, similar groups, recall some of their basic properties, and show that they satisfy the Farrell-Jones conjectures.

We follow a slightly unorthodox path to define (self-)similar groups: we first give their contracting covers, and then simply say that the group itself is the faithful quotient of the cover. This, of course, defines uniquely the self-similar group $G$ in question: it is the quotient of its universal contracting cover $F$ by the normal subgroup $K_{\infty} \triangleleft F$.

We denote by $\left\langle\left\langle g_{1}, \ldots, g_{d}\right\rangle\right\rangle \sigma$ an element of the wreath product $G \imath d$, with $\sigma$ written as a product of disjoint cycles.

4.1. The Alëshin and Grigorchuk groups. The Alëshin-Grigorchuk group is obtained as follows. Set

$$
F=\left\langle a, b, c, d \mid a^{2}, b^{2}, c^{2}, d^{2}, b c d\right\rangle=C_{2} *\left(C_{2} \times C_{2}\right),
$$


and define $\phi: F \rightarrow F>2$ by

$$
\phi(a)=\langle\langle 1,1\rangle\rangle(1,2), \quad \phi(b)=\langle\langle a, c\rangle\rangle, \quad \phi(c)=\langle\langle a, d\rangle\rangle, \quad \phi(d)=\langle\langle 1, b\rangle\rangle .
$$

Let $G$ be the faithful self-similar quotient of $F$.

This group (up to finite index) was first considered in [1], providing a "tangible" example of infinite, finitely generated, torsion group (the first examples of groups with these properties are due to Golod [17]. Sushchansky [27] also constructed selfsimilar $p$-groups for odd prime $p$ ). Grigorchuk proved in [18] that its word growth is strictly between polynomial and exponential, and in [20] that it is amenable, but not elementary amenable. It is contracting, with nucleus $\{1, a, b, c, d\}$.

Since $F$ is CAT $(0)$, as a free product of finite groups, $G$ satisfies the Farrell-Jones conjectures by Proposition 1 .

More elaborate examples have also been constructed by Grigorchuk [19]. Fix an infinite sequence $\omega=\omega_{0} \omega_{1} \ldots$ of epimorphisms $\left(C_{2} \times C_{2}\right) \cong\langle b, c, d\rangle \rightarrow$ $\langle a\rangle \cong C_{2}$, and assume that $\omega$ contains infinitely many of each of the three possible epimorphisms. Define homomorphisms $\phi_{n}: F \rightarrow F<2$ for all $n \geq 0$ by

$$
\phi_{n}(a)=\langle\langle 1,1\rangle\rangle(1,2), \quad \phi_{n}(x)=\left\langle\left\langle\omega_{n}(x), x\right\rangle\right\rangle \text { for } x \in\{b, c, d\} .
$$

Let $G_{\omega}$ be the faithful similar quotient of $F$ using this similarity structure.

Again, $G_{\omega}$ is contracting with nucleus $N_{n}=\{1, a, b, c, d\}$ for all $n \in \mathbb{N}$, so all such groups satisfy the Farrell-Jones conjectures. There are uncountably many such groups, and they are all torsion 2-groups of intermediate word growth.

4.2. The Gupta-Sidki groups. The Gupta-Sidki groups are obtained as follows. Choose a prime $p \geq 3$, set

$$
F=\left\langle a, t \mid a^{p}, t^{p}\right\rangle=C_{p} * C_{p},
$$

and define $\phi: F \rightarrow F<p$ by

$$
\phi(a)=\langle\langle 1, \ldots, 1\rangle\rangle(1, \ldots, p), \quad \phi(t)=\left\langle\left\langle a, a^{-1}, 1, \ldots, 1, t\right\rangle\right\rangle .
$$

Let $G$ be the faithful self-similar quotient of $F$.

These groups are shown in [22] to be infinite, finitely generated torsion $p$-groups.

Since $F$ is CAT( $(0)$, as a free product of finite groups, $G$ satisfies the Farrell-Jones conjectures by Proposition 1 .

4.3. Bounded groups. Assume that $G$ is a self-similar group, and that, for every $g \in G$, there exists a bound $B \in \mathbb{N}$ such that, for all $n \in \mathbb{N}$, there are at most $B$ non-trivial entries in $\phi^{n}(g)$. Note that it suffices to check this property for the generators of $G$; and that it holds for the generators of the Grigorchuk group with $B=2$, and those of the Gupta-Sidki groups with $B=3$. 
It is then known (see [12]) that $G$ is contracting. More precisely, $G$ is isomorphic to a subgroup of a self-similar group of very special type (see [9]). Fix an integer $d \geq 2$, set

$$
F=\mathfrak{\subseteq}_{d} *\left(\Im_{d} 2 \Im_{d}-1\right),
$$

and define $\phi: F \rightarrow F<d$ by

$$
\phi(\sigma)=\langle\langle 1, \ldots, 1\rangle\rangle \sigma, \quad \phi\left(g:=\left\langle\left\langle f_{1}, \ldots, f_{d-1}\right\rangle\right\rangle \tau\right)=\left\langle\left\langle f_{1}, \ldots, f_{d-1}, g\right\rangle\right\rangle \tau .
$$

Since $F$ is CAT( 0$)$, as a free product of finite groups, $G$ satisfies the Farrell-Jones conjectures by Proposition 1.

Note that the faithful quotient of $F$ is amenable; this is how [9] shows that all bounded self-similar groups are amenable.

4.4. Dynamics. Let $f$ be a branched covering of a topological space $\mathcal{M}$; this means there is an open dense subset $\mathcal{M}_{0} \subset \mathcal{M}$ and a covering $f: \mathcal{M}_{0} \rightarrow \mathcal{M}$. We assume $f$ has finite degree $d$. Let $P_{f}$ denote the post-critical locus of $f$ :

$$
P_{f}=\bigcup_{n \geq 1} f^{n}\left(\mathcal{M} \backslash \mathcal{M}_{0}\right) .
$$

Assume finally that $\mathcal{M} \backslash P_{f}$ is path-connected. Choose a basepoint $* \in \mathcal{M} \backslash P_{f}$, and for each $x \in f^{-1}(*)$ choose an arc $\ell_{x}$ from $*$ to $x$ in $\mathcal{M} \backslash P_{f}$. Number also $f^{-1}(*)$ as $\left\{x_{1}, \ldots, x_{d}\right\}$.

These data define a self-similar group as follows. It is again defined via a cover, $F:=\pi_{1}\left(\mathcal{M} \backslash P_{f}, *\right)$. Consider $\gamma \in F$. For each $x_{i} \in f^{-1}(*)$, let $\gamma_{i}$ denote the unique $f$-lift of $\gamma$ that starts at $x_{i}$, and let it end at $x_{\pi(i)} \in f^{-1}(*)$. Define then $\phi: F \rightarrow F<d$ by

$$
\phi(\gamma)=\left\langle\left\langle\ell_{\pi(1)}^{-1} \gamma_{1} \ell_{1}, \ldots, \ell_{\pi(d)}^{-1} \gamma_{d} \ell_{d}\right\rangle\right\rangle \pi
$$

with the product of paths given by concatenation in the right-to-left order.

If $\mathcal{M}$ is in fact a locally simply connected metric space, and $f$ is uniformly expanding (meaning there exists $\lambda>1$ such that $d(f x, f y)>\lambda d(x, y)$ whenever $d(x, y)$ is sufficiently small), then $F$ is contracting.

This applies in particular to $\mathcal{M}$ a complex manifold and $f$ a holomorphic map (which is then expanding for the Kobayashi metric).

The special case $\mathcal{M}=\mathbb{C}$ and $f$ a degree-2 polynomial has been extensively studied in [10]. The cover $F$ is a free group, so this provides more examples of groups satisfying the Farrell-Jones conjectures. One important such example, associated with the map $f(z)=z^{2}-1$, has been studied in [21] and [11]; it is amenable, orderable, of exponential growth, and residually poly- $\mathbb{Z}$.

Other examples, on higher-dimensional manifolds, have been considered by Koch et al. [24], [14]. There, the universal contracting cover is the sphere braid group. 


\section{Conclusion}

We have shown that if a counter-example to the Farrell-Jones conjectures exists in the class of (self-)similar groups, it will not be an easy matter to find it.

For one thing, with very few exceptions, non-contracting self-similar groups are intractable (it required considerable effort to prove that the elementary example of [2] is a free group!)

For another, calculations in a contracting self-similar groups are usually reduced to calculations in a finitely presented group, in which one may manipulate words. It would be surprising that the Farrell-Jones conjectures fail for a self-similar group, yet be unsettled for its cover.

Since the Farrell-Jones conjectures are not settled for the sphere braid group, we have, at the present, no argument to check the Farrell-Jones conjectures on the faithful self-similar quotient of the braid groups that arise in this manner.

Let $G$ be a self-similar group, and let $e \in \mathbb{N}$ be given. Assume that, for every $g \in G$, there exists a bound $B \in \mathbb{N}$ such that, for all $n \in \mathbb{N}$, there are at most $B n^{e}$ non-trivial entries in $\phi^{n}(g)$. Then $G$ is said to be of polynomial activity growth of degree $e$; see [26], who proves that such groups do not contain free subgroups.

It is then known [3] that $G$ embeds, possibly for larger $d$, in a specific group $P(d, e)$ of polynomial activity growth, defined by its cover as follows. Set $\Sigma_{-1}=\mathfrak{\Im}_{d}$ and $\Sigma_{i}=\Sigma_{i-1}<\mathfrak{S}_{d-1}$ for $i=0, \ldots, e$; set

$$
F=\Sigma_{-1} * \cdots * \Sigma_{e}
$$

and define $\phi: F \rightarrow F<d$ by

$$
\begin{aligned}
& \phi(\sigma)=\langle\langle 1, \ldots, 1\rangle\rangle \sigma, \\
& \phi(g)=\left\langle\left\langle f_{1}, \ldots, f_{d-1}, g\right\rangle\right\rangle \tau \text { for } g=\left\langle\left\langle f_{1}, \ldots, f_{d-1}\right\rangle\right\rangle \tau \in \Sigma_{i}, i \geq 0 .
\end{aligned}
$$

These are non-contracting self-similar groups if $e \geq 1$; for $e \leq 1$, the faithful quotient is amenable [11], [3], while amenability of the faithful quotient is open for larger $e$.

The arguments in [26] show that the nucleus $N$ of $P(d, e)$, while infinite, admits a partial well ordering, such that every $g \in N$ has the form $g \in \Sigma_{-1}$ or $\phi(g)=$ $\left\langle\left\langle g_{1}, \ldots, g_{d-1}, g\right\rangle\right\rangle$ with $g_{i}<g$ for all $i \in\{1, \ldots, d-1\}$. Presumably this means that arguments similar to those given here show that $P(d, e)$, and therefore all its subgroups, satisfy the Farrell-Jones conjectures.

It has been conjectured by Nekrashevych that all contracting self-similar groups are amenable; although no conclusive link has been established between amenability and the Farrell-Jones conjectures.

At the other extreme of contracting self-similar groups lie bireversible groups. These are self-similar groups $(G, \phi)$ such that the map $G \times\{1, \ldots, d\} \rightarrow G \times$ $\{1, \ldots, d\}$, given by $(g, i) \mapsto\left(g_{i}, \sigma(i)\right)$ if $\phi(g)=\left\langle\left\langle g_{1}, \ldots, g_{d}\right\rangle\right\rangle \sigma$, is a bijection. They are related to the infinite simple groups constructed in [16], [15]. They would seem like a natural class in which to look at counterexamples, though all examples studied up to now are lattices in virtually connected Lie groups. 


\section{References}

[1] S. V. Aleshin, Finite automata and Burnside's problem for periodic groups. Mat. Zametki 11 (1972), 319-328; English transl. Math. Notes 11 (1972), 199-203. Zbl 0253.20049 MR 0301107

[2] S. V. Aleshin, A free group of finite automata. Vestnik Moskov. Univ. Ser. I Mat. Mekh. 1983 (1983), No. 4, 12-14; English transl. Moscow Univ. Math. Bull. 38 (1983), No. 4, 10-13. Zbl 0513.68044 MR 713968

[3] G. Amir, O. Angel, and B. Virag, Amenability of linear-activity automaton groups. J. Eur. Math. Soc. (JEMS), to appear; Preprint 2009. arXiv:0905.2007

[4] A. Bartels, F. T. Farrell, and W. Lück, The Farrell-Jones Conjecture for cocompact lattices in virtually connected Lie groups. Preprint 2011. arXiv:1101.0469

[5] A. Bartels and W. Lück, On crossed product rings with twisted involutions, their module categories and $L$-theory. In Cohomology of groups and algebraic K-theory, Adv. Lect. Math. (ALM) 12, Internat. Press, Somerville, MA, 2010, 1-54. Zbl 1205.19006 MR 2655174

[6] A. Bartels and W. Lück, The Borel conjecture for hyperbolic and CAT(0) groups. Ann. of Math. (2) 175 (2012), 631-689. Zbl 06025000 MR 2993750

[7] A. Bartels, W. Lück, and H. Reich, On the Farrell-Jones conjecture and its applications. J. Topol. 1 (2008), 57-86. Zbl 1141.19002 MR 2365652

[8] L. Bartholdi, R. I. Grigorchuk, and Z. Šuniḱ, Branch groups. In Handbook of algebra. Vol. 3, North-Holland, Amsterdam 2003, 989-1112. Zbl 02156384 MR 2035113

[9] L. Bartholdi, V. A. Kaimanovich, and V. V. Nekrashevych, On amenability of automata groups. Duke Math. J. 154 (2010), 575-598. Zbl 05816151 MR 2730578

[10] L. Bartholdi and V. V. Nekrashevych, Iterated monodromy groups of quadratic polynomials. I. Groups Geom. Dyn. 2 (2008), 309-336. Zbl 1153.37379 MR 2415302

[11] L. Bartholdi and B. Virág, Amenability via random walks. Duke Math. J. 130 (2005), 39-56. Zbl 1104.43002 MR 2176547

[12] E. Bondarenko and V. Nekrashevych, Post-critically finite self-similar groups. Algebra Discrete Math. 2003 (2003), no. 4, 21-32. Zbl 1068.20028 MR 2070400

[13] M. R. Bridson and A. Haefliger, Metric spaces of non-positive curvature. Grundlehren Math. Wiss. 319, Springer-Verlag, Berlin 1999. Zbl 0988.53001 MR 1744486

[14] X. Buff, A. Epstein, S. Koch, and K. Pilgrim, On Thurston's pullback map. In Complex dynamics. Families and friends. A K Peters, Wellesley, MA, 2009, 561-583. Zbl 1180.37065 MR 2508269

[15] M. Burger and S. Mozes, Finitely presented simple groups and products of trees. C. $R$. Acad. Sci. Paris Sér. I Math. 324 (1997), 747-752. Zbl 0966.20013 MR 1446574

[16] M. Burger and S. Mozes, Groups acting on trees: from local to global structure. Inst. Hautes Études Sci. Publ. Math. 92 (2000), 113-150. Zbl 1007.22012 MR 1839488

[17] E. S. Golod, On nil-algebras and finitely approximable p-groups. Izv. Akad. Nauk SSSR Ser. Mat. 28 (1964), 273-276; English transl. Amer. Math. Soc. Transl. (2) 48, Amer. Math. Soc., Providence, RI, 1965, 103-106. Zbl 0215.39202 MR 0161878 
[18] R. I. Grigorchuk, On the Milnor problem of group growth. Dokl. Akad. Nauk SSSR 271 (1983), 30-33; English transl. Soviet Math. Dokl. 28 (1983), 23-26. Zbl 0547.20025 MR 712546

[19] R. I. Grigorchuk, Degrees of growth of finitely generated groups, and the theory of invariant means. Izv. Akad. Nauk SSSR Ser. Mat. 48 (1984), 939-985; English transl. Math. USSR-Izv. f25 (1985), 259-300. Zbl 0583.20023 MR 0764305

[20] R. I. Grigorchuk, An example of a finitely presented amenable group not belonging to the class EG. Mat. Sb. 189 (1998), 79-100; English transl. Sb. Math. 189 (1998), 75-95. Zbl 0931.43003 MR 1616436

[21] R. I. Grigorchuk and A. Żuk, Spectral properties of a torsion-free weakly branch group defined by a three state automaton. In Computational and statistical group theory (Las Vegas, NV/Hoboken, NJ, 2001), Contemp. Math. 298, Amer. Math. Soc., Providence, RI, 2002, 57-82. Zbl 1057.60045 MR 1929716

[22] N. Gupta and S. Sidki, On the Burnside problem for periodic groups. Math. Z. 182 (1983), 385-388. Zbl 0513.20024 MR 696534

[23] L. Kaloujnine and M. Krasner, Le produit complet des groupes de permutations et le problème d'extension des groupes. C. R. Acad. Sci. Paris 227 (1948), 806-808. Zbl 0038.16203 MR 0027758

[24] S. C. Koch, Teichmüller theory and applications to endomorphisms of $\mathbb{P}^{n}$. Ph.D. thesis, Université de Provence, Marseille 2007.

[25] V. Nekrashevych, Self-similar groups. Math. Surveys Monogr. 117, Amer. Math. Soc., Providence, RI, 2005. Zbl 1087.20032 MR 2162164

[26] S. Sidki, Finite automata of polynomial growth do not generate a free group. Geom. Dedicata 108 (2004), 193-204. Zbl 1075.20011 MR 2112674

[27] V. I. Suščans'ki1̆, Periodic $p$-groups of permutations and the unrestricted Burnside problem. Dokl. Akad. Nauk SSSR 247 (1979), 557-561; English transl. Soviet Math. Dokl. 20 (1979), 766-770. Zbl 0428.20023 MR 0545692

[28] C. Wegner, The $K$-theoretic Farrell-Jones conjecture for CAT(0)-groups. Proc. Amer. Math. Soc. 140 (2012), 779-793. Zbl 1240.19003 MR 2869063

Received July 29, 2011; revised September 21, 2011

L. Bartholdi, Mathematisches Institut, Georg-August Universität zu Göttingen, Bunsenstraße 3-5, 37073 Göttingen, Germany

E-mail: laurent.bartholdi@gmail.com 